\title{
Gaussian Mixture Filter for Multipath Assisted Positioning
}

\author{
Markus Ulmschneider, German Aerospace Center (DLR), Germany, markus.ulmschneider@dlr.de \\ Christian Gentner, German Aerospace Center (DLR), Germany, christian.gentner@dlr.de \\ Ramsey Faragher, Focal Point Positioning, United Kingdom, ramsey@fppnt.com \\ Thomas Jost, German Aerospace Center (DLR), thomas.jost@dlr.de
}

\section{BIOGRAPHIES}

Markus Ulmschneider studied communications and computer engineering at the University of Ulm, Germany, from where he received his Bachelor's degree in 2011 and his Master's degree in 2014. In 2014, he joined the Institute of Communications and Navigation of the German Aerospace Center (DLR), Germany, where he is part of the scientific staff of the Mobile Radio Transmission group. His main research interests include multipath assisted positioning as well as multi-sensor localization and tracking techniques.

Christian Gentner studied electrical engineering at the University of Applied Science in Ravensburg, with the main topic communication technology and received his Dipl.-Ing. (BA) degree in 2006. During this study he received practical experiences at Rohde \& Schwarz in Munich. He continued his study at the University of Ulm until 2009, where he received his M.Sc. degree. He is currently working towards the Ph.D. degree at the Institute of Communications and Navigation of the German Aerospace Center (DLR). His current research focuses on multipath assisted positioning.

Ramsey Faragher is an expert in GNSS-denied positioning, sensor fusion, and machine-learning. $\mathrm{He}$ is the founder and CEO of Focal Point Positioning, a Fellow of the Royal Institute of Navigation, and a Bye Fellow of Queens College, Cambridge. Previously he has held research positions at the University of Cambridge and was a principal scientist at BAE Systems where he developed the NAVSOP opportunistic positioning suite and other GNSS-denied tracking technologies.

Thomas Jost received a Diploma degree (FH) 2001 in Electrical Engineering from University of Applied Science Wiesbaden, Germany, and a Diploma degree in 2003 in Electrical Engineering and Information Technology from Technical University of Darmstadt, Germany. In 2013, he received his $\mathrm{PhD}$ from University of Vigo, Spain. From 2003 to 2006 he held a research assistant position in the Signal Processing Group at TU Darmstadt. Since 2006 he is a member of the scientific staff of the Institute of Communications and Navigation at the German Aerospace Center.
Abstract-Navigation in global navigation satellite system denied areas such as urban canyons or indoors has aroused large interest due to the recent growth of location aware services. In these scenarios, multipath assisted positioning schemes are promising due to a rich multipath propagation. Instead of trying to combat multipath, multipath assisted positioning approaches make use of multipath components arriving at a receiver that is to be located. In more detail, multipath components arriving at the receiver via different paths are regarded as pure line-ofsight signals from virtual transmitters. In general, the number of transmitters might be large, and their location may be unknown. The underlying estimation problem, i.e., estimating the positions of the receiver and the physical and virtual transmitters, tends to be very costly in computational terms. Within this paper, we present a Rao-Blackwellization approach to tackle the computational burden. The receiver location is tracked using a particle filter, while the probability density functions of the transmitter states are represented by Gaussian mixture models, whose parameters are estimated using cubature Kalman filters.

\section{INTRODUCTION}

While global navigation satellite systems (GNSSs) perform well under good view-to-sky conditions, they are known to show weak performance in urban or indoor scenarios due to a low received signal power, multipath propagation, and shadowing [1]. In these situations, wireless navigation and positioning technologies using signals of opportunity ( $\mathrm{SoO}$ ) have aroused more and more research interest. However, multipath propagation has been considered a curse in urban and indoor scenarios using wireless systems, as it biases range estimates and hence decreases the positioning accuracy. Standard methods to overcome the multipath problem are based on the estimation of the channel impulse response (CIR) at the receiver. These methods try to mitigate the influence of multipath components (MPCs) on the line-of-sight (LoS) path to increase the accuracy.

Instead of trying to mitigate and combat MPCs, the idea of multipath assisted positioning is contrary: MPCs may actually be exploited for positioning. Each of the signal components, no matter if arriving at the receiver via the LoS path or a different propagation path, can be regarded as a SoO from a transmitter in a pure LoS condition to the receiver. If the signal component arrives via the LoS path, the corresponding transmitter is an actual physical transmitter, otherwise, we may cal 1 the transmitter a virtual transmitter. Both the physical and 
the virtual transmitters can now be used for positioning at the receiver.

Fundamental limits and theoretical results on multipath assisted positioning have been presented in [2]. Some approaches using multipath assisted positioning assume the physical and virtual transmitter positions to be known in advance, for example in form of a floorplan in indoor [3] or in radar [4] applications. In a general setting, however, the positions of the virtual and possibly also of the physical transmitters are unknown. Therefore, with the Channel-SLAM algorithm, an approach has been presented in [5] and [6] that does not rely on a-priori knowledge of the transmitter positions. Instead, their locations are estimated jointly with the receiver position using a recursive Bayesian estimation approach. Each transmitter state probability density function (PDF) is initialized based on the first time of arrival (ToA) measurement for the corresponding signal component at a mobile terminal carrying the receiver. Again, each MPC is treated as being sent from a virtual transmitter in a pure LoS condition to the receiver. Since we assume only ToA measurements to be available, and due to possible clock offsets of the transmitters, the initialization of a transmitter PDF is performed with a high uncertainty.

Estimating the entire state, i.e., the mobile terminal position and the location of all transmitters, is expensive in terms of computational complexity. While the Kalman filter provides an optimal and efficient solution to such problems, it can not be applied due to the nonlinearity of the underlying ToA measurement model. Other recursive Bayesian estimation methods, such as particle filters, suffer from the curse of dimensionality. Though, Rao-Blackwellization techniques tackle that problem and have been applied successfully to simultaneous localization and mapping (SLAM) problems.

Within this paper we propose a new recursive Bayesian estimation approach that is based on the idea of RaoBlackwellization: Assuming independence between the ToA measurements associated to each transmitter enables estimating their states independently. We use a particle filter that estimates the mobile terminal position. For each particle representing a hypotheses of the mobile terminal state, we estimate the state of each transmitter by means of a Gaussian mixture filter, where we regard each Gaussian component as a weighted hypotheses for the state of the respective transmitter. We use a cubature Kalman filter (CKF) presented in [7] to estimate the parameters of the Gaussian components. The idea of the CKF is to approximate the integrals in the Bayesian estimation process by a closed form cubature rule.

The rest of this paper is organized as follows. Section II introduces the underlying concepts and methods. In Section III, we introduce the estimation problem at hand and our solution to it. We present simulations in order to assess the performance of our algorithm in Section IV. Section V concludes the paper.

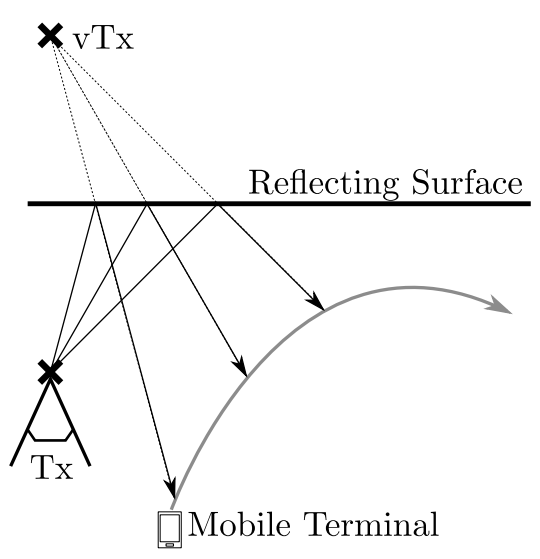

Fig. 1. The signals emitted by the transmitter Tx are reflected by the surface and received by a mobile terminal. From the mobile terminal perspective, as it moves along its track, the reflected signals seem to be emerging from the point vTx.

\section{System Model}

\section{A. Virtual Transmitters}

The idea of virtual transmitters is illustrated in Fig. 1: The physical transmitter Tx broadcasts a signal that is received by the mobile terminal equipped with a receiver. As depicted, one signal component arrives at the mobile terminal via a reflection at the straight, reflecting surface, i.e., as a MPC. From the mobile terminal point of view, this signal may be regarded as being sent from the virtual transmitter vTx in a pure LoS condition. The position of the virtual transmitter is the position of the physical transmitter Tx mirrored at the surface. As the mobile terminal moves along its trajectory, the position of the virtual transmitter is constant if the environment and the physical transmitter are static, and hence it might be used for positioning purposes. In addition, the virtual transmitter is inherently synchronized to the physical transmitter. Depending on the amount of multipath propagation in the scenario, the number of virtual transmitters might allow to obtain a unique positioning solution even if only single physical transmitter is present. Furthermore, the physical transmitter does not even have to be in LoS to the receiver.

The concept of virtual transmitters can be transferred from a reflecting surface to a punctual scatterer. Then, the position of the virtual transmitter is equal to the position of the scatterer. However, for the punctual scatterer case, the virtual transmitters have a clock offset to the physical transmitter. Also, a generalization to the case where the signal is reflected and/or scattered multiple times can be made in a straightforward manner [5].

\section{B. Recursive Bayesian Estimation}

Recursive Bayesian estimation allows in general for recursively estimating a PDF of a state vector $\mathbf{x}$ [8]. It is assumed that the state vector follows a first order hidden Markov model, and can not be observed directly. The evolution of $\mathrm{x}$ over time is modeled as 


$$
\mathbf{x}_{k}=\mathbf{f}_{k}\left(\mathbf{x}_{k-1}, \mathbf{v}_{k-1}\right)
$$

where $\mathbf{x}_{k}$ and $\mathbf{x}_{k-1}$ are the state vector at time steps $k$ respectively $k-1, \mathbf{f}_{k}(\cdot)$ is a known function, and $\mathbf{v}_{k}$ is the realization of a process noise sequence at time step $k$. Eq. (1) describes the so-called movement or state transition model of the process. In the same way, measurements that are taken can be related to the state vector as

$$
\mathbf{z}_{k}=\mathbf{h}_{k}\left(\mathbf{x}_{k}, \mathbf{n}_{k}\right)
$$

where $\mathbf{z}_{k}$ is the measurement, $\mathbf{h}_{k}(\cdot)$ is a known function, and $\mathbf{n}_{k}$ is a realization of a measurement noise sequence at time step $k$. The goal is now to estimate the state at all time steps 0 to $k$, i.e., $\mathbf{x}_{0: k}$, given the $k$ conducted measurements $\mathbf{z}_{1: k}$. This is, to estimate the posterior PDF $\mathrm{p}\left(\mathbf{x}_{0: k} \mid \mathbf{z}_{1: k}\right)$. This problem can be approached recursively in two stages, namely prediction and update stage. In the prediction stage, a-priori information on the state transition is utilized if available. In the update stage, the prediction is corrected based on measurements. If the movement and measurement model, i.e., the functions $\mathbf{f}_{k}(\cdot)$ and $\mathbf{h}_{k}(\cdot)$ in Eq. (1) respectively Eq. (2), are linear, and if the noise samples $\mathbf{v}_{k}$ and $\mathbf{n}_{k}$ are uncorrelated and drawn from Gaussian distributions, an optimal estimator to the problem is the Kalman filter.

If the physical transmitters and the environment are static, the virtual transmitters are static as well. Our goal is to track the mobile terminal's position and velocity in two dimensions, and to estimate the locations of the transmitters as well as their clock offsets to the receiver. Hence, we define the mobile terminal state vector at a time step $k$ as

$$
\mathbf{x}_{\mathrm{MT}, k}=\left[\begin{array}{llll}
x_{\mathrm{MT}} & y_{\mathrm{MT}} & v_{x, \mathrm{MT}} & v_{y, \mathrm{MT}}
\end{array}\right]^{T},
$$

and the state vector of the $j^{\text {th }}$ of the physical and virtual transmitters as

$$
\mathbf{x}_{\mathrm{TX}, k}^{<j>}=\left[\begin{array}{lll}
x_{\mathrm{TX}, j} & y_{\mathrm{TX}, j} & \tau_{\mathrm{TX}, j}
\end{array}\right]^{T},
$$

where $\tau_{\mathrm{TX}, j}$ represents the transmitter's clock offset. Given the number of transmitters being $N_{\mathrm{TX}}$, the entire state vector at time step $k$ is given by

$$
\begin{aligned}
\mathbf{x}_{k} & =\left[\begin{array}{lll}
\mathbf{x}_{\mathrm{MT}, k}^{T} & \mathbf{x}_{\mathrm{TX}, k}^{T}
\end{array}\right]^{T} \\
& =\left[\begin{array}{llll}
\mathbf{x}_{\mathrm{MT}, k}{ }^{T} & \mathbf{x}_{\mathrm{TX}, k}^{<0>T} & \ldots & \mathbf{x}_{\mathrm{TX}, k}^{<N_{\mathrm{TX}}-1>T}
\end{array}\right]^{T} .
\end{aligned}
$$

\section{Gaussian Mixture Models}

The Gaussian mixture model used here represents the PDF of a transmitter state by a sum of $N_{\mathrm{CKF}}$ Gaussian PDFs, or Gaussian components. Each of the Gaussian components is described by a mean and a covariance matrix, and has a weight associated to it. The weight of the $\ell^{\text {th }}$ component for the $j^{\text {th }}$ transmitter at time step $k$ is denoted by $w_{k}^{<j, \ell>}$, and the sum of all $N_{\mathrm{CKF}}$ weights for one transmitter equals one. Representing the PDF for the $j^{\text {th }}$ transmitter at time step $k$ as a Gaussian mixture, we have [9]

$$
\mathrm{p}\left(\mathbf{x}_{\mathrm{TX}, k}^{<j>} \mid \mathbf{z}_{1: k}^{<j>}\right)=\sum_{\ell=1}^{N_{\mathrm{CKF}}} w_{k}^{<j, \ell>} \mathcal{N}\left(\mathbf{x}_{\mathrm{TX}, k}^{<j>} ; \hat{\mathbf{x}}_{\mathrm{TX}, k \mid k}^{<j, \ell>}, \mathbf{P}_{k \mid k}^{<j, \ell>}\right),
$$

where $\hat{\mathbf{x}}_{\mathrm{TX}, k \mid k}^{<j, \ell>}$ and $\mathbf{P}_{k \mid k}^{<j, \ell>}$ are the mean and the covariance matrix of the $\ell^{\text {th }}$ Gaussian component of the $j^{\text {th }}$ transmitter, respectively, and $\mathbf{z}_{1: k}^{<j>}$ are the measurements from time step 1 to $k$ for the $j^{\text {th }}$ transmitter. The function $\mathcal{N}(\mathbf{x} ; \mu, \mathbf{C})$ describes a Gaussian PDF with mean $\mu$ and covariance matrix $\mathbf{C}$.

\section{Cubature Kalman Filter}

In our system model, the ToA measurement model between the transmitters and the receiver is nonlinear in the state. Some of the integrals involved in the estimation process are therefore intractable or do not have a closed-form solution. We propose to use a CKF to estimate the transmitter state vectors. The CKF is a variant of the unscented Kalman filter (UKF) originally presented in [10]. It numerically approximates those integrals by exploiting their special structure, as the integrands are the product of an arbitrary function $\mathbf{g}(\cdot)$ and a Gaussian PDF $\mathcal{N}(\mathbf{x} ; \mathbf{0}, \mathbf{I})$. The integral can then be approximated by a thirddegree spherical-radial cubature rule, which results in a general form in

$$
\int_{\mathbb{R}^{n}} \mathbf{g}(\mathbf{x}) \mathcal{N}(\mathbf{x} ; \mathbf{0}, \mathbf{I}) \mathrm{d} \mathbf{x} \approx \frac{1}{2 n} \sum_{t=1}^{2 n} \mathbf{g}\left(\xi_{t}\right),
$$

where $n$ is the number of states in the state space,

$$
\xi_{t}=\sqrt{n} Q_{t},
$$

and $Q_{t}$ is the $t^{\text {th }}$ cubature point from the cubature points set $\mathbf{Q}$. For further details on the CKF, we refer the reader to [7].

\section{Proposed Algorithm}

\section{A. Estimation Problem}

The state vector $\mathbf{x}_{k}$ that is to be estimated can grow huge depending on the number of visible transmitters, i.e., signal components arriving at the receiver. To make the estimation process more efficient, we follow a Rao-Blackwellization approach and split the state space into the mobile terminal state space and the transmitter state space. For the posterior density, we hence have

$$
\begin{aligned}
\mathrm{p}\left(\mathbf{x}_{0: k} \mid \mathbf{z}_{1: k}\right) & =\mathrm{p}\left(\mathbf{x}_{\mathrm{TX}, 0: k}, \mathbf{x}_{\mathrm{MT}, 0: k} \mid \mathbf{z}_{1: k}\right) \\
& =\mathrm{p}\left(\mathbf{x}_{\mathrm{TX}, 0: k} \mid \mathbf{x}_{\mathrm{MT}, 0: k}, \mathbf{z}_{1: k}\right) \mathrm{p}\left(\mathbf{x}_{\mathrm{MT}, 0: k} \mid \mathbf{z}_{1: k}\right)
\end{aligned}
$$

We use a sequential importance resampling (SIR) particle filter [11] to estimate the mobile terminal state $\mathbf{x}_{\mathrm{MT}, k}$. For each of the particles representing a hypotheses of the the mobile terminal state, we estimate the transmitter states given the mobile terminal state vector. This idea is reflected in the first 


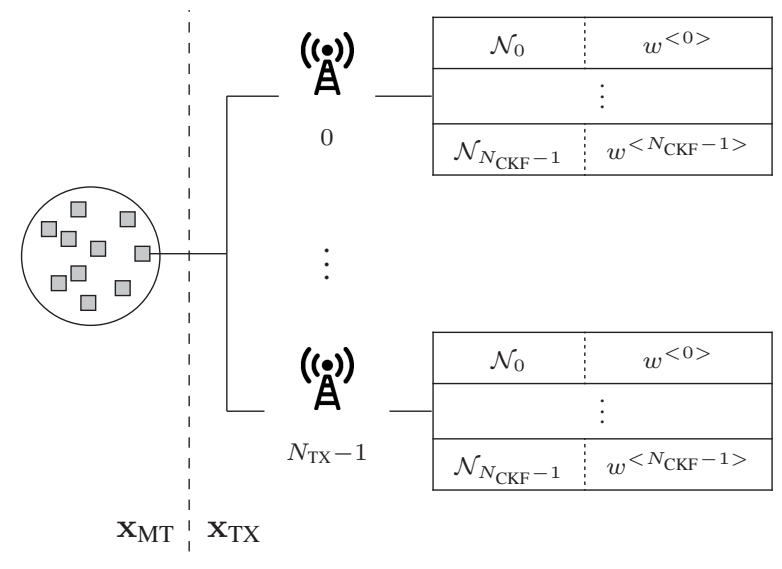

Fig. 2. The mobile terminal state $\mathbf{x}_{\mathrm{MT}}$ is tracked using a particle filter. For each particle, the $N_{\mathrm{TX}}$ transmitter states are estimated independently from each other using a Gaussian sum filter, where each of the $N_{\mathrm{CKF}}$ hypotheses is represented by a Gaussian distribution $\mathcal{N}_{\ell}$ with an associated weight $w^{<\ell>}$.

factor of the right hand side on the second line in Eq. (3): it is the posterior PDF for the transmitter states given the state of the mobile terminal and the measurements up to time step $k$, multiplied by the PDF for the actual mobile terminal state given the measurements.

The state transition models for the single transmitter states are independent from each other. In addition, we assume independence between the ToA measurements that are obtained for the single transmitters. Hence, we can factorize the likelihood at time step $k$ as

$$
\mathrm{p}\left(\mathbf{z}_{k} \mid \mathbf{x}_{\mathrm{MT}, k}, \mathbf{x}_{\mathrm{TX}, k}\right)=\prod_{j=1}^{N_{\mathrm{TX}}} \mathrm{p}\left(\mathbf{z}_{k}^{<j>} \mid \mathbf{x}_{\mathrm{MT}, k}, \mathbf{x}_{\mathrm{TX}, k}^{<j>}\right),
$$

where $\mathbf{z}_{k}^{<j>}$ is the measurement for the $j^{\text {th }}$ transmitter at time step $k$. For each mobile terminal particle, we can now estimate each transmitter state independently using a Gaussian mixture filter. Each weighted Gaussian component of the Gaussian mixture represents one hypotheses for the transmitter state.

We obtain a ToA measurement for each visible transmitter at time step $k$, and model the ToA measurement for the $j^{\text {th }}$ transmitter as

$$
\hat{\mathbf{z}}_{k}^{<j>}=\frac{1}{c_{0}}\left\|\mathbf{p}_{\mathrm{MT}, \mathrm{k}}-\mathbf{p}_{\mathrm{TX}, \mathrm{k}}^{<j>}\right\|+\tau_{\mathrm{TX}, j}+\mathbf{n}_{k},
$$

where $\mathbf{p}_{\mathrm{MT}, \mathrm{k}}$ and $\mathbf{p}_{\mathrm{TX}, \mathrm{k}}^{<j>}$ are the locations of the mobile terminal and the $j^{\text {th }}$ transmitter, respectively, at time step $k$, $\|\cdot\|$ denotes the Euclidean norm, and $c_{0}$ is the speed of light. To estimate the parameters of the Gaussian components of the transmitters' states, the nonlinearity in the measurement model does not allow to use Kalman filters. We choose a CKF to cope with the nonlinearity. Based on the incoming measurements, the weights of the Gaussian components and the weight of the particles in the particle filter are updated at every time step.

Fig. 2 illustrates the filtering structure: We use a particle

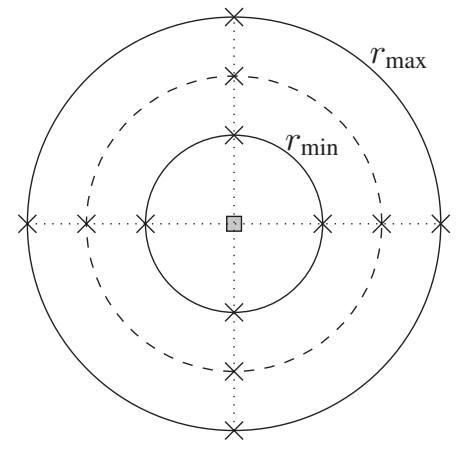

Fig. 3. Example for the spatial initialization of twelve Gaussian components representing hypotheses for one transmitter. The components are indicated by crosses, and they are located on three circles around a mobile terminal particle located in the center.

filter to track the mobile terminal's state vector. Each gray square in the circle on the left hand side represents one particle, i.e., one hypothesis in the mobile terminal state space. We have $N_{\text {TX }}$ transmitters whose states are estimated independently from each other for each mobile terminal particle, as exemplarily drawn for the rightmost particle. In turn, each transmitter's state is represented by a Gaussian mixture, a sum of weighted Gaussian distributions $\mathcal{N}_{\ell}$ with an associated weight $w^{<\ell>}$. The vertical dashed line in Fig. 2 differentiates between the mobile terminal state space representation on the left, and the transmitters' state space representation on the right.

\section{B. Initialization and Time Progress}

We assume to have no prior information on the true transmitter states upon their initialization. We initialize each transmitter state PDF after the first ToA for this transmitter has been measured. Given only one ToA measurement and an unknown clock offset, the uncertainty for each transmitter state at initialization is very high. Hence, we need a lot of hypotheses, i.e., Gaussian components in the mixture model. The initial transmitter hypotheses are spread spatially such that the coordinates in the mean of the corresponding component are located on circles around the current mobile terminal position hypotheses. The radius of the innermost circle is chosen as some $r_{\min }$, the radius of the outermost circle, $r_{\max }$, is equal to the first ToA measurement multiplied by the speed of light. The radii of the circles in between are chosen such that they divide the interval between $r_{\min }$ and $r_{\max }$ equally. On each of the circles, the means of the components are evenly spread. Having initialized the components spatially, their clock offset is chosen such that it matches the first ToA measurement. Hence, the clock offsets for the Gaussian components on the outermost circle is zero. An example for twelve Gaussian components is shown in Fig. 3. The gray square in the center represents the particle, i.e., the hypotheses for the mobile terminal position. The crosses represent the position coordinates in the mean of the Gaussian components for one transmitter, located on three circles with equidistant radii. On each circle, we have four Gaussian components. 
TABLE I

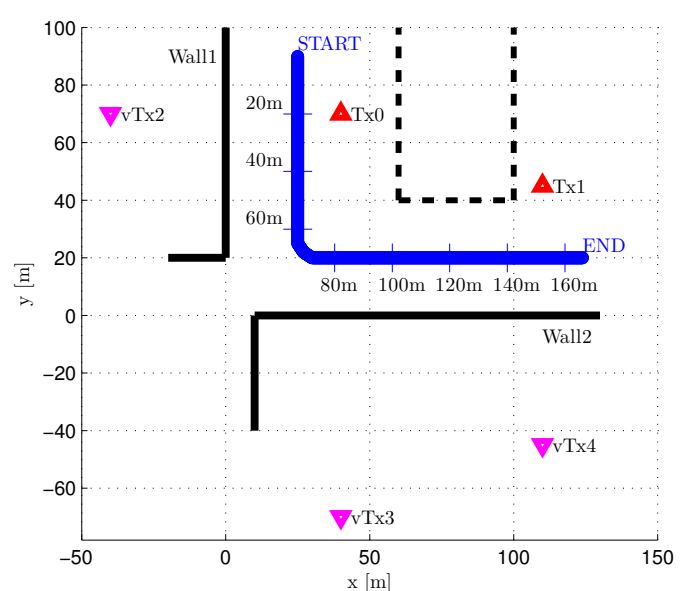

Fig. 4. A simple urban scenario with physical transmitters $\mathrm{Tx} 0$ and $\mathrm{Tx} 1$, reflecting walls Wall1 and Wall2, and corresponding virtual transmitters vTx2, vTx3, and vTx4. The receiver track is depicted in blue with the start and end positions marked as START respectively END. The traveled distance is marked for every $20 \mathrm{~m}$. Black, dashed lines are non-reflecting, but blocking walls.

The weights associated to the $N_{\mathrm{CKF}}$ single components are initialized equally as $1 / N_{\mathrm{CKF}}$. They are updated and normalized at every time step based on the incoming ToA measurements. As the mobile terminal moves through the scenario, many of the initial hypotheses for the transmitters' states will not match the measurements. If their weight falls below a threshold, we discard the corresponding Gaussian components from the mixture model. Hence, the number of Gaussian components in the transmitter state PDFs might change over time and be different for each transmitter of each mobile terminal particle.

\section{Simulations}

\section{A. Simulation Scenario}

For verifying our estimation approach, we performed simulations exploiting the multipath propagation in a simple urban scenario, which is depicted in Fig. 4. Solid black lines represent walls reflecting the transmit signals, whereas black, dashed lines are non-reflecting but blocking walls. We have two physical transmitters, Tx0 and Tx1, that are represented by red upward triangles. Knowing the environment, we model two virtual transmitters for Tx0. These are vTx2, arising due to reflections of signals originating from $\mathrm{Tx} 0$ at the wall to the left (marked as Wall1), and vTx3, arising due to reflections of signals from Tx0 at the lower wall (marked as Wall2). The position of vTx2 respectively vTx3 is the position of the physical transmitter Tx0 mirrored at Wall1 respectively Wall2. Similarly, we have one virtual transmitter vTx4 for the physical transmitter Tx1 due to reflections of signals from Tx 1 at Wall2, and the position of vTx4 is the position of Tx 1 mirrored at Wall2. The virtual transmitters are depicted by magenta colored downward triangles.

The mobile terminal carrying a receiver moves on a track represented by the blue line with a constant velocity of $1.8 \mathrm{~m} / \mathrm{s}$.
OBJECTS AND THEIR COORDINATES IN THE SIMULATION SCENARIO.

\begin{tabular}{ll}
\hline Object & Coordinates \\
\hline Tx0 & $(40,70)$ \\
Tx1 & $(110,45)$ \\
vTx2 & $(-40,70)$ \\
vTx3 & $(40,-70)$ \\
vTx4 & $(110,-45)$ \\
receiver START & $(25,90)$ \\
receiver END & $(124,20)$ \\
\hline
\end{tabular}

The start and end positions are indicated by the labels START respectively END. Not all transmitters are visible at any mobile terminal position due to blocking by the walls. Every $700 \mathrm{~ms}$, the receiver takes a ToA measurement for the currently available MPCs. The exact coordinates of the transmitters and the start and end position of the mobile terminal are listed in Table I. We simulate an inertial measurement unit (IMU) at the mobile terminal to avoid the ambiguity when the mobile terminal turns left after approx. $64 \mathrm{~m}$ of traveled distance. However, we only make use of the IMU heading information, since we have experienced that the heading information even of low-cost IMUs tends to be very reliable without much processing, while the IMU speed information tends to drift considerably. For simulating the IMU heading data, we use the true heading data of the mobile terminal and add white Gaussian noise.

At the beginning of the mobile terminal track, the view to the transmitter Tx1 is blocked (see Fig. 4). After approx. $100 \mathrm{~m}$ of traveled distance, Tx 1 becomes visible and is initialized at the receiver. Having traveled another $15 \mathrm{~m}$, the LoS to transmitter Tx0 is lost, and this physical transmitter cannot be used anymore. Though, the virtual transmitters vTx2 and vTx3, which arise due to the reflections of the signals emitted from Tx0, can still be used. After a traveled distance of approx. $141 \mathrm{~m}$, vTx3 cannot be used any further, since the signal traveling from Tx0 to the mobile terminal via Wall2 is blocked.

We assume the starting position and the initial direction of the mobile terminal to be known in order to define a local coordinate system. For its tracking, 1000 particles are initialized normally distributed around the true mobile terminal position with a variance of $1 \mathrm{~m}^{2}$. For the mobile terminal state transition model, we make use of heading information from the simulated IMU. For the mobile terminal speed, a random walk model is implemented.

The ToA measurements in the simulation are the true ToA values with additive white Gaussian noise. They are taken at the receiver for every transmitter that is visible at a certain time step. We assume ultra-wideband (UWB) signals, and hence that the signal components arriving at the receiver can be well resolved. This means that ToA the measurements for the single physical and virtual transmitters are taken independently from each other. 


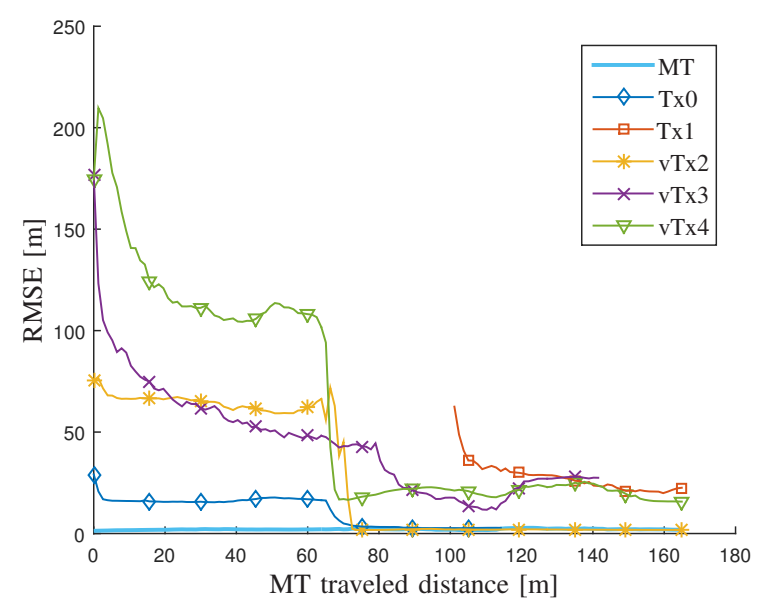

Fig. 5. The RMSE for the mobile terminal (MT) and the transmitters is plotted against the mobile terminal traveled distance.

No a-priori knowledge on the transmitter positions and clock offsets is assumed. When the first ToA for a transmitter is measured, the transmitter is initialized as described in Subsection III-B. We choose $r_{\min }=10 \mathrm{~m}$ and place 768 Gaussian components on twelve circles, resulting in 64 components per circle. The threshold for pruning Gaussian components equals $10^{-15}$.

\section{B. Simulation Results}

Fig. 5 shows the simulation results. The root mean square error (RMSE) for the mobile terminal position and the locations of the physical and virtual transmitters is plotted against the distance the mobile terminal has traveled.

The RMSE for the mobile terminal position is always below $3 \mathrm{~m}$ throughout the mobile terminal motion. After initialization, the error is very high for the transmitters since no information on them is given. Already after the first meters, many hypotheses for the transmitters are pruned as the weights of the Gaussian components fall below the threshold. Hence, the RMSE for the transmitters decreases. Though, as the mobile terminal walks in a straight line in the beginning, there is the ambiguity on which side of the line each transmitter is located, and this ambiguity flattens the RMSE curves for the transmitters in that region. When the mobile terminal takes a turn to the right after a traveled distance of approx. $64 \mathrm{~m}$, using heading information from the simulated IMU, the ambiguity can be resolved, and the RMSE decreases during and right after the turn. At a traveled distance of approx. $100 \mathrm{~m}$, the physical transmitter Tx 1 becomes visible for the first time, and is initialized. Since the mobile terminal moves on a straight track after this initialization, the ambiguity for Tx1 cannot be resolved, and hence the RMSE curve for Tx1 flattens out. Having completed the track, the final values for the RMSE are given in Table II.

While the RMSE for transmitters Tx0 and vTx2 drop down below $3 \mathrm{~m}$ respectively $2 \mathrm{~m}$ during the mobile terminal motion, the errors for the transmitters vTx3 and vTx4 flatten out
TABLE II

RMSE IN METERS AFTER COMPLETING THE TRACK.

\begin{tabular}{ll}
\hline Object & RMSE $[\mathrm{m}]$ \\
\hline mobile terminal & 1.85 \\
Tx0 & 2.60 \\
Tx1 & 22.56 \\
vTx2 & 1.77 \\
vTx3 & 27.65 \\
vTx4 & 15.73 \\
\hline
\end{tabular}

towards the end of the track, and stay on a relatively high value. The reason is that the mobile terminal track does not fully exploit the geometry of the scenario. The mobile terminal receives LoS signals from Tx0 from a wide range of angles while moving on its track, which is why many initial hypotheses for the state vector of $\mathrm{Tx} 0$ are pruned. In contrast, signals from vTx3 are received only from a limited range of angles. Hence, more initial hypotheses are kept alive and prevent the RMSE for Tx3 from decreasing. In the same way, the good RMSE performance for vTx2, and the relatively high RMSE for vTx4 can be explained.

\section{Conclusion}

We presented an new estimation scheme for multipath assisted positioning in this paper. The transmitters' states are represented by Gaussian mixture models. The single Gaussian densities represent hypotheses for the transmitter states, and they are pruned if their weight falls below a threshold. We use CKFs to estimate these Gaussian densities, and a particle filter for estimating the mobile terminal state. A urban scenario was used for simulations to verify our algorithm.

\section{REFERENCES}

[1] N. Agarwal, J. Basch, P. Beckmann, P. Bharti, S. Bloebaum, S. Casadei, A. Chou, P. Enge, W. Fong, N. Hathi, W. Mann, A. Sahai, J. Stone, J. Tsitsiklis, and B. Van Roy, "Algorithms for gps operation indoors and downtown," GPS Solutions, vol. 6, no. 3, pp. 149-160, 2002.

[2] Y. Shen and M. Win, "On the use of multipath geometry for wideband cooperative localization," in IEEE Globecom, Nov. 2009, pp. 1-6.

[3] P. Meissner and K. Witrisal, "Multipath-assisted single-anchor indoor localization in an office environment," in Systems, Signals and Image Processing (IWSSIP), 2012 19th International Conference on, Apr. 2012, pp. 22-25.

[4] P. Setlur, G. Smith, F. Ahmad, and M. Amin, "Target localization with a single sensor via multipath exploitation," Aerospace and Electronic Systems, IEEE Transactions on, vol. 48, no. 3, pp. 1996-2014, Jul. 2012.

[5] C. Gentner, T. Jost, and A. Dammann, "Indoor positioning using time difference of arrival between multipath components," in IEEE IPIN, Montbeliard, France, Oct. 2013.

[6] C. Gentner, R. Pöhlmann, M. Ulmschneider, T. Jost, and A. Dammann, "Multipath assisted positioning for pedestrians," in Proceedings of the ION GNSS+, Tampa, FL, USA, Sep. 2015.

[7] I. Arasaratnam and S. Haykin, "Cubature kalman filters," Automatic Control, IEEE Transactions on, vol. 54, no. 6, pp. 1254-1269, June 2009.

[8] S. Kay, Fundamentals of Statistical Signal Processing: Estimation Theory, ser. Fundamentals of Statistical Signal Processing. PrenticeHall PTR, 1998.

[9] B. Anderson and J. Moore, Optimal Filtering, ser. Dover Books on Electrical Engineering. Dover Publications, 2012. 
[10] S. J. Julier, J. K. Uhlmann, and H. F. Durrant-Whyte, "A new approach for filtering nonlinear systems," in American Control Conference, Proceedings of the, vol. 3, Jun 1995, pp. 1628-1632.

[11] M. Arulampalam, S. Maskell, N. Gordon, and T. Clapp, "A tutorial on particle filters for online nonlinear/non-gaussian bayesian tracking," Signal Processing, IEEE Transactions on, vol. 50, no. 2, pp. 174-188,

Feb. 2002 